\title{
Chapter 4 \\ Higher Education Spaces and Protracted \\ Displacement: How Learner-Centered \\ Pedagogies and Human-Centered Design \\ Can Unleash Refugee Innovation
}

\author{
Barbara Moser-Mercer, Erin Hayba and Joshua Goldsmith
}

\subsection{Introduction}

Today, the world is seeing the largest number of refugees and displaced persons in history, with a total of 65.5 million forcibly displaced and over 22 million refugees in 2016 (UNHCR 2016). The average conflict lasts 10 years, and families are displaced for an average of 20 years. Against a backdrop of competing humanitarian needs and priorities, the education response is often limited, leaving entire generations uneducated, developmentally disadvantaged, and unprepared to contribute to their society's recovery (UNHCR 2011).

Traditionally, Education in Emergencies responses have focused on primary education; higher education opportunities have often been perceived as a luxury. Current statistics on refugee access to education confirm this ongoing trend: $50 \%$ of refugee children access primary education, $22 \%$ secondary education, and only $1 \%$ higher education. Children and youth are particularly vulnerable to losing their right to education, a basic human right that is enshrined in the 1989 Convention on the Rights of the Child and the 1951 Refugee Convention, and is essential to the exercise of many other human rights. In 2015, the United Nations adopted the Sustainable Development Goals, thereby broadening the education mandate to include lifelong learning.

While refugee youth have extremely limited options in conflict and crisis zones (Sheehy 2015), rapid advances in technology, online learning and open educational resources (OERs) have laid the foundations for making higher education opportunities accessible for refugee youth.

B. Moser-Mercer $(\varangle) \cdot$ E. Hayba $\cdot$ J. Goldsmith

University of Geneva, InZone, Geneva, Switzerland

e-mail: Barbara.Moser@unige.ch

E. Hayba

e-mail: erinhayba@gmail.com

J. Goldsmith

e-mail: Goldsmith.joshua@gmail.com

(C) The Author(s) 2018

S. Hostettler et al. (eds.), Technologies for Development,

https://doi.org/10.1007/978-3-319-91068-0_4 
Education fosters innovation and entrepreneurial skills that are important for employability, economic activity, and job creation-elements that are critical for stability during times of reconstruction and for longer term sustainable development. If refugees and internally displaced persons receive a quality education while in exile, they are more likely to develop the necessary skills to make use of the existing economic, social, and political systems in their host communities as well as upon returning home.

\subsection{Background}

Over the years, InZone-a Higher Education in Emergencies (HEiE) actor whose mission is to design, develop and scientifically validate HEiE models that respect humanitarian principles-has identified key HEiE challenges: access to education, quality of educational programs, relevance of programs, and management of programs in the field and remotely.

During an initial mapping exercise of higher education providers active in the refugee education space, quantitative data was collected using an online questionnaire and disaggregated. This data presented a snapshot of existing programs in terms of several key features, such as size, graduation rates, funding, scholarship availability, accreditation, types of courses, degrees and fields of study, learning spaces, and use of technology. In the second phase of the research, in-depth interviews were conducted with educators responsible for running a number of such higher education programs. Interviews were analyzed using computer-assisted qualitative data analysis (CAQDAS) programs and a series of inductive codes and constructs which emerged holistically from the data (cf. Bryman 2004; Corbin and Strauss 2007; Merriam 2009).

Cross-cutting themes that emerged during this mapping exercise included cultural dimensions, intellectual and learning cultures, the development of twentyfirst-century skills, peer-to-peer and collaborative learning, pedagogical models that integrate ICT and connectivity, responsible partnerships, information sharing and communication, leveraging refugees' skills and ingenuity, and preventing brain and knowledge drain.

When designing programs for fragile contexts, most HEiE providers have aimed to facilitate equal access to programs. Nevertheless, a series of physical, intellectual, financial and legal factors have hampered this access, including insufficient infrastructure and connectivity, the length and cost of travel to the learning center, insecurity, a lack of ICT skills, language barriers, religious and cultural factors, course costs, and a lack of needed legal documentation, particularly education credentials, to access higher education. Providers have adopted a wide range of innovative solutions to surmount these challenges.

Though HEiE programs attempt to survey the contexts in which they will be operating before launching their programs, it generally takes several editions of a course to develop approaches which dovetail with the reality on the ground. HEiE 
providers often offer programs which they believe would be of use to refugee learners, such as teacher training courses, yet these models, which are generally incubated in the Global North, must be adapted so that they are accessible for and meaningful to refugee learners and provide options for learning pathways.

One of the main questions to emerge from these reflections is just how refugee learners can learn in such a context. How can learners communicate with institutions of higher learning and with organizations willing to support refugee access to higher education? How can learners share their needs across languages and cultures, including learning cultures, particularly if the gaps in understanding are too deep and too wide? How do providers foster cultural and linguistic ownership and ensure that courses are culturally and linguistically relevant?

The main challenge for higher education providers entails escaping the lure of tried-and-true higher education models. How can we avoid the attractiveness of the concept of humanitarian assistance as a way to "provide"? How can we rise to the challenge and recognize that the greatest successes shall be found in a participatory approach that engages and empowers beneficiaries to co-design solutions and make learning a two-way process? Indeed, the true beneficiaries of all Higher Education in Emergencies initiatives must be refugee learners and their communities, since learning is both an individual and a social activity, and the knowledge and skills refugee learners acquire should also help advance their communities.

\subsubsection{Disruptive Higher Education in Fragile Contexts: Digital Ecosystems and Open Educational Resources (OERs)}

Even in traditional Higher Education (HE) models, education is not synonymous with taking a course or pursuing a course of study, whether in a lecture hall, a seminar room or on a learning portal. Education includes knowledge and skill acquisition, instruction, debate, application of acquired expertise, critical inquiry, cultural expression, and transmission to other members of the community and society.

Refugee youth enrolled in Higher Education programs also seek learning environments that challenge them to learn and grow beyond the textbook or classroom. While the liberty to think and challenge ideas and opinions is often the norm in universities, such space is limited or nonexistent in refugee contexts. In times of crisis, insecurity, fear, and destruction, youth are greatly affected and have few opportunities and even less space for free and open thinking. Furthermore, refugee youth are often too old to qualify for most educational programs and too young to have much experience or stability to help them through such difficult times (Evans and Forte 2013). Living in dangerous conflict and crisis areas poses numerous challenges for refugee youth, including the threats of armed groups and tight legal restrictions on employment and freedom of movement (Burde 2014). 
Higher Education thrives in larger spaces that offer diverse inputs and encourage free expression, allowing learners to combine free navigation of learning resources with more structured tutoring and mentoring approaches. Unorthodox learning pathways unleash creativity; interrupted learning pathways, however, are hurdles. Protracted displacement is the biggest such hurdle, a major stumbling block to satisfying intellectual curiosity and leading a dignified life. This is due to the feeling of helplessness and insecurity that can compound feelings of despair and hopelessness over years of displacement with limited or no educational or economic opportunities.

Open Educational Resources (OERs), and more specifically Massive Open Online Courses (MOOCs), have disrupted Higher Education in the Global North by challenging traditional assumptions and practices about teaching and learning, most notably by putting the learner at the center of the design and using technology to enable the learning process. As such, MOOCs have acted as a catalyst for change and have prompted many educators to rethink what education should look like today and how we can best design it.

Through our ongoing research into the challenges of refugee access to higher education (Moser-Mercer 2016), we have learned that despite extraordinary growth in ICTs, access to information in fragile contexts remains constrained by connectivity, language, and cultural barriers. Furthermore, information per se does not constitute education, and this is particularly true for learners living in fragility. Higher Education in Emergencies cannot simply replicate traditional Higher Education models. Indeed, HEiE must unlock the innovation potential of its "users" in an uncertain environment by operating flexibly while showing tolerance for ambiguity, adaptability, and equifinality, i.e., researching multiple pathways to reach its objectives (Ries 2011; Blank 2013; Bloom and Betts 2014).

This perspective motivated InZone to undertake the MOOC Multi-Center Study (Moser-Mercer 2016). This project aimed to engage in a finer grain analysis of the multiple factors that contribute to the development of optimal learning environments in fragile contexts. These factors must be responsive to refugee learners' needs, respect humanitarian principles, and create opportunities for charting new learning pathways in situations of conflict, crisis, and protracted emergencies.

The study built on an earlier case study (Moser-Mercer 2014) in an effort to strengthen the evidence base for HEiE by including a variety of fragile learning contexts. These differed with respect to the setting - camp or urban refugee settings; language-multilingual with English as vehicular host country language or predominantly monolingual with Arabic as vehicular host country language; and gender diversity. Even after enlarging the learner pool, expanding into different urban- and camp-based refugee settings, ensuring diversity in the participant pool and incorporating refugees as participant researchers, the earlier case study results were largely confirmed.

The study used an off-the-shelf MOOC offered consecutively as a session-based and on-demand course in English. It integrated as variables (a) on-site and virtual tutoring and mentoring support in select settings, enlisted the partnership of NGOs and international organizations with an education mandate and local presence, and (b) two geographic regions - the Horn of Africa and the Middle East-and two 
vehicular languages-English and Arabic. The research aim was to increase our understanding of the variables which mediate successful learning outcomes for all refugee learners. Especially when it came to the variable of tutor support, the qualitative analysis generated particularly useful results designed to inform the adaptation of OERs in general, and MOOCs in particular, to fragile contexts. In fact, through quantitative analysis of on-site tutoring support the latter emerged as the single most decisive variable to boost learner success, particularly for female refugee learners in refugee camp settings. The study carefully documented the differential use of social media and learning technologies and concluded that fragile learning contexts make use of different modes of modern communication and that choices are usually dictated by connectivity, accessibility, and financial affordances.

This confirmed the relevance of the Technology-Access-Matrix, designed by InZone to support all of its online and blended higher education programming (InZone 2015). This matrix contends that higher education courses for learners in fragile contexts should be designed to integrate a carefully planned progression regarding the use of learning technologies and communication media in an effort to provide pedagogically sound incidental learning opportunities. This contrasts with the more traditional approach to HEiE, whereby ICT courses are offered prior to launching content-rich and engaging higher education programs, thus artificially separating the acquisition of ICT skills from the actual skill of learning. This approach compartmentalizes skills that together are essential for supporting learning, making it more difficult for learners to understand their respective relevance and hampering their required cognitive integration. Skill and knowledge acquisition are co-determined by learners' capacity, learning opportunities, motivation, and engagement. In challenging learning contexts, integrative learning pedagogies are vital to building successful learning strategies.

Support courses, such as language enhancement or ICT courses, often do not elicit the kind of engagement from learners in general, and refugee learners in particular need to maintain motivation in the face of contextual adversity. This spans a wide range of challenges, including insufficient, irregular access to learning resources that are not explicitly made available by course providers in a durable format, but which learners are expected to access; and language and cultural barriers that represent cognitive challenges that are not easily resolved in a virtual learning environment.

The MOOC Multi-Center Study offers rich qualitative and stringent quantitative data to inform course providers about learner support requirements. These need to be integrated into course planning if large numbers of refugee learners are to succeed and remain motivated to continue their learning journey. While this study did not specifically address the question of credentialing OERs and allowing learners engaged in non-formal higher education to obtain regular academic credit for their work, the different requirements for session-based and on-demand versions of OERs and the way learners navigated these provide important information as to how such credentialing may have to be approached in the future. Of particular note are the issues related to hard deadlines, peer-assessed learning activities, learning ethics-including the interpretation of the concept of plagiarism across different cultures-and free-loading. In the refugee context, learning goes global. Nevertheless, humanitarian 
principles, humanitarian accountability, and guidelines for education in emergencies have recently been developed by the Connected Learning in Crisis Consortium ( http://connectedlearning4refugees.com) to ensure that all voices are heard and all cultures, including intellectual cultures, are respected.

Learning from and designing with higher education learners thus emerges as the optimal approach to HEiE, as it puts the refugee learner in the center of the design process, builds on bottom-up processes of evidence-generation, leverages humanitarian partnerships in an effort to meet humanitarian principles and guidelines, and thus promotes participatory innovation. Innovation is already part of the humanitarian system. It is driven by a demand for new models and rapid technological change (Bloom and Betts 2014). User-linked and user-led or "indigenous innovation" is by definition inclusive and thus has the potential to foster ingenuity among youth living in fragility contexts.

Since OERs deliver knowledge, encourage experimentation with information, and invite adaptation to local contexts and languages, they are both "adoptable" and "ownable." Such innovation is often termed frugal, grassroots, or BoP (bottom-ofpyramid); HEiE actors consistently point out the challenges of designing for, with and by users, yet unanimously agree that such an approach opens up many innovative possibilities for not only finding solutions to problems but also ensuring that these solutions are compatible with the local context and thus have a higher chance of successful diffusion (Smith et al. 2013). As Bloom and Betts (2014, p. 28) aptly state, "...researchers and entrepreneurs from outside the traditional humanitarian agencies benefit from collaborating with end-users and agencies to define problem statements and designs." Although bottom-up innovation in fragile contexts is subject to humanitarian, legal, economic, and social constraints, there is considerable space for engagement and "innovation spaces," which can be physical or virtual spaces for learning, interaction, and sharing ideas and resources. These spaces are enabled by a digital ecosystem that supports HE models offering quality education opportunities and livelihoods and encourages safe, non-physical ways of resolving conflict, thereby addressing a major humanitarian challenge.

Learning and designing from and with our learners is indeed the bottom-up approach that has continually informed InZone's research: When learners reflect on their challenges on a regular basis, they not only improve their meta-cognitive skills but are ultimately empowered to be the agents of the change they wish to bring about. Research and pedagogy are thus intimately intertwined; every study carried out in fragile contexts should also incorporate a pedagogical agenda. This will discourage traditional research approaches-where refugees are merely the subjects of research and subsequent solutions and models are imposed on them-and encourage a participatory approach that engages refugees as active research contributors and designers of their own spaces. 


\subsubsection{Designing HE Spaces in Settings of Protracted Displacement: The Case of the Kakuma InZone HE Space}

InZone launched higher education courses in Kakuma Refugee Camp (located on the border between Kenya and South Sudan) in 2012, and in Dadaab Refugee Camp (located on the border between Kenya and Somalia) in 2013. Within the larger methodological framework of a case study, and for the purposes of this article, the authors present an incremental empirical approach to designing HE spaces in fragile contexts while observing humanitarian principles. The Kakuma InZone HE Space represents a case study that pools qualitative and quantitative data collected across different refugee camp settings and explores the potential of collaborative pedagogies to address protracted conflict.

While written within a non-refugee context, Radcliffe's (2009) statement on designing education spaces can be readily transposed to fragile contexts. According to Radcliffe, a learning space should motivate learners and promote learning as an activity; it should support individual, collaborative, and formal practice and provide a personalized and inclusive environment. Furthermore, it should be flexible, adapting to changing needs and contextual challenges and to the chosen pedagogical approach. The more challenging the context, the more important the design and the greater the degree of flexibility that is required to let users adapt different parts of the space to support different dimensions of their learning.

Radcliffe's pedagogy-technology-space framework rests on a number of important assumptions about designing optimal learning environments. First, pedagogy is enabled by space, which in turn encourages pedagogical innovation. Second, space embeds technology, which in turn expands the space and opens it up to entire communities of learners, both locally and globally. Third, pedagogy benefits from technology as an enabler, while technological innovation is enhanced by pedagogical considerations. Higher education spaces for refugee learners should thus be flexible, to accommodate current and evolving pedagogies; future-proofed, to enable reallocation and reconfiguration of space; bold, to look beyond tried and tested technologies and pedagogies; creative, to energize and inspire learners and tutors; supportive, to develop the potential of all learners; and enterprising, so that each space can support different purposes.

In order to implement InZone's collaborative, learner-centered pedagogy—which is embedded in its framework of skill acquisition and expertise-InZone is piloting the design of a Higher Education Space for Refugees in Kakuma Refugee camp (InZone HE Space Kakuma). The design incorporates twenty-first-century approaches to technology-supported learning in an extremely challenging learning context, a refugee camp. The design is bold in that it looks beyond traditional approaches to higher education by integrating both formal and non-formal learning opportunities; access to credit-bearing and diploma-granting higher education programs is blended with non-formal higher education programming. This includes MOOCs, which are offered in both English and French, as well as on-site work- 
shops that leverage the potential of the arts-dance, movement, applied drama, storytelling, and creative writing - to allow learners to be creative and to unleash their creative potential to co-design their learning environment and amplify the knowledge acquired through higher education opportunities through sharing it with the entire refugee community. It is also future-proofed, by allowing for reallocation of space-the Kakuma InZone Student Café hosts student discussion groups, community performances, and learning groups-and can expand to other parts of the camp, since the Café is geographically separate from the InZone Learning Hub. The design is flexible, adapting to complementary pedagogical approaches and enabling both individual and collaborative learning; it is enterprising, in that each space can support different learning activities; and most of all, it is supportive, continuously encouraging learners to creatively use their wider learning space and thereby encouraging all $\mathrm{HE}$ refugee learners to develop their potential to the fullest.

The concept of an HE space in fragile contexts thus covers and enables the entire range of learning possibilities. It begins with structured spaces for traditional faceto-face teaching, online and blended learning in the InZone Learning Hub, where learners can either work individually or collaboratively with or without tutor support and thus with more or less pedagogical structure. It encompasses debates in the InZone Café, operated by Ethiopian refugee learners who ensure that traditional coffee ceremonies are passed on to learners in exile. It extends all the way to less structured learning spaces, such as the French library, and ultimately includes the home environment. Learning is therefore not confined to a specific location or to a specific environment. Rather, learning happens in a range of contexts, some of which are more structured than others. Incidental and formal learning are both equally encouraged. The HE space transitions easily across contexts, as do refugee learners, who often hold multiple jobs in order to eke out a living in the camp. Most importantly, though, the Kakuma InZone Higher Education Space is always open to redesign and configurable to the needs of different higher education programming. It is open for collaboration with other higher education providers in the camp. Consequently, it contributes to establishing a community of higher education learners, who are equipping themselves with twenty-first-century skills and thereby contributing to making their communities conflict-resilient as they actively search for peaceful alternatives to the wars they have left behind. Most importantly, the Kakuma InZone Higher Education Space is refugee managed through a team of refugee learners and InZone alumni, rather than through an implementing partner, as is traditional in humanitarian and development projects. Skills and competencies acquired through higher education thus support employability and secure livelihoods, thereby empowering refugees to design and redesign the learning space in careful synergy with their community. 


\subsubsection{Learner-Centered Pedagogies and Human-Centered Design in Support of SDG 4 and EFA}

In 2015, the Sustainable Development Goals (SDGs) were adopted by the United Nations to expand upon the Millennium Development Goals which concluded that same year. SDG 4 focuses on education and reads, "Ensure inclusive and quality education for all and promote lifelong learning." This SDG redefines and shifts the approach to education set out in the previous MDG on education: learning is now considered a lifelong process, and is not limited to primary education. According to the most recent UNESCO Global Monitoring Report on Education for All (EFA) in 2014, great progress was made in recent years towards reaching the targets laid out in the MDGs, particularly for universal primary education. Progress towards targets in secondary education access was also made. Yet in areas of conflict, the number of out-of-school children has increased (UNESCO 2015).

Humanitarian education programs have typically focused on children, particularly those enrolled in primary education, as educational programming in humanitarian contexts is decidedly reliant and linked to international policies (Zeus 2011). Yet, with the new shift towards lifelong learning embedded in SDG 4, humanitarian education programs will need to be more holistic, broadening their approach to youth and higher education. International humanitarian and development policies play an instrumental role in determining education programming on the ground in conflict and crisis settings, setting the stage for institutions of higher learning to engage as humanitarian actors.

The global targets laid out in the SDGs refer to a broad mandate of deliverables by the year 2030. Among these, specific mention is given to tertiary education; increasing the number of youth and adults with skills for employment and entrepreneurship; educating children and youth in vulnerable situations; and promoting peace and appreciation of diversity. These targets, while ambitious, will require innovative solutions and changes to current educational programming in emergency contexts. More importantly, the learners in these communities should be active contributors to the design and implementation of such programs. Human-centered design will thus emerge as a critical aspect of this process, which can be further promoted through learner-centered pedagogies that have been an integral part of the InZone approach and which prepare refugee learners for lifelong learning journeys.

In designing and producing OERs with other HEiE actors in the North and the South, InZone has taken its lead from refugee learners themselves, engaging them in the design process and supporting bottom-up innovation. Evidence from the implementation of OER-driven courses informs the creation of new resources, particularly with respect to how to design "the last mile" to optimally support learning outcomes. Higher Education programming for fragile contexts should be field-proofed and codesigned by learners before it aspires to scale. To meet the objectives of SDG 4 and EFA, we must listen to beneficiaries. By engaging them in the design of both content and delivery, we will foster communication across languages and cultures in the field, ensure that learning materials are contextualized and access to learning is guaranteed, 
and pilot promising solutions together before going to scale. In fragile contexts, beneficiary input to all phases of Higher Education provision is thus essential and needs to be integrated into all SDG-4 programming and funding schemes.

\subsection{Conclusions}

This paper has analyzed the contribution of Open Educational Resources to building twenty-first-century skills; noted the value of tutoring and mentoring models for optimal learning outcomes, learner retention and the provision of language and subject matter support; and explored the technologies, including learning technologies, that best mediate higher level learning in fragile contexts. Variables such as sustainability, operability, equal access, cultural and linguistic ownership, livelihoods, and context relevance were used to analyze available evidence in an effort to inform optimal design and scalability of such learning spaces, as well as their potential use in migrant refugee contexts.

We conclude that human-centered design is central to both pedagogical and technological development in fragile contexts. In light of the fact that protracted displacement settings can differ considerably from one another, there is no substitute for adopting a bottom-up approach and developing learning spaces that allow for both intellectual and artistic expression. Traditional HE programs are usually geared to specific degrees, still offer little flexibility, and do not cater adequately yet to alternative learning pathways. Online education platforms offer a wide range of learning options and a variety of pedagogical approaches. Such innovative tools and programs increase access to learning and offer great potential for higher education; however, such initiatives may face resistance from traditional university programs, particularly due to challenges regarding accreditation, recognition of degrees, and credit transfers (Hullinger 2015).

OERs have the potential to inspire non-traditional learners and encourage an explore-and-develop approach rather than a listen-and-reproduce mentality. They are thus a gateway that allows refugees to unleash their innovation potential. We emphasize the importance of refugee ownership and empowerment as vectors for ensuring the sustainability of higher level learning in HE spaces in fragile contexts, analyze the potential of ubiquitous learning supported in broadly conceived and flexible learning spaces for fostering creativity and innovation, and encourage the inclusion of innovative and learner-centered pedagogical approaches that support such learning in the larger framework of Education for All and Sustainable Development Goal 4. 


\section{References}

Blank, S. (2013). Why the lean start-up changes everything. Harvard Business Review, 91, 3-9.

Bloom, L. \& Betts, A. (2014). Humanitarian innovation: The state of the art. Occasional Paper Series UN-OCHA, November 2014/009. Available from http://www.unocha.org and http://www. reliefweb.int.

Bryman, A. (2004). Social research methods. New York: Oxford University Press.

Burde, D. (2014). Schools for conflict or for peace in Afghanistan. New York: Columbia University Press.

Corbin, J., \& Strauss, A. (2007). Basics of qualitative research: Techniques and procedures for developing grounded theory (3rd ed.). Thousand Oaks, CA: Sage.

Evans, R., \& Lo Forte, C. (2013). A global review: UNHCR's engagement with displaced youth. Geneva: Switzerland.

Hullinger, J. (2015). This is the future of college. Last accessed November 12, 2017 from http:// www.fastcompany.com/3046299/the-new-rules-of-work/this-is-the-future-of-college.

InZone. (2015). Technology-access-matrix. Unpublished internal InZone report. Geneva: University of Geneva/InZone.

Merriam, S. B. (2009). Qualitative research: A guide to design and implementation. San Francisco: Jossey-Bass.

Moser-Mercer, B. (2014). MOOCs in fragile contexts. In U. Kress \& C. D. Kloos (Eds.), Proceedings of the European MOOC Stakeholder Summit 2014 (EPFL Lausanne) (pp. 114-121). ISBN 97884-8294-689-4. Available from www.openeducationeuropa.eu.

Moser-Mercer, B. (2016). MOOC multi-center-study 2015. Unpublished Internal InZone report. Geneva: University of Geneva/InZone.

Radcliffe, D. (2009). A pedagogy-space-technology (PST) framework for designing and evaluating learning spaces. In D. Radcliffe, H. Wilson, D. Powell, \& B. Tibbetts (Eds.), Learning spaces in higher education: Positive outcomes by design (pp. 9-16). Brisbane: The University of Queensland.

Ries, E. (2011). The lean startup: How today's entrepreneurs use continuous innovation to create radically successful businesses. New York: Randomhouse.

Sheehy, I. (2015). Tertiary education in conflict and crisis: The new landscape. Lecture. Last accessed November 11, 2017 from https://mediaserver.unige.ch/fichiers/view/89797.

Smith, A., Fressoli, M., \& Thomas, H. (2013). Grassroots innovation movements: challenges and contributions. Journal of Cleaner Production, 63, 114-124. https://doi.org/10.1016/j.jclepro. 2012.12.025.

UNESCO. (2015). EFA global monitoring report 2015: Education for all 2000-2015: Achievements and challenges. Paris: UNESCO.

UNHCR. (2011). Refugee education: A global review. Geneva: UNHCR.

UNHCR. (2016). UNHCR statistics portal. Geneva, Switzerland. Last accessed November 13, 2017 from http://www.unhcr.org/figures-at-a-glance.html.

Zeus, B. (2011). Exploring barriers to higher education in protracted refugee situations: The case of Burmese refugees in Thailand. Journal of Refugee Studies, 24(2), 256-276. https://doi.org/10. 1093/jrs/fer011. 
Open Access This chapter is licensed under the terms of the Creative Commons Attribution 4.0 International License (http://creativecommons.org/licenses/by/4.0/), which permits use, sharing, adaptation, distribution and reproduction in any medium or format, as long as you give appropriate credit to the original author(s) and the source, provide a link to the Creative Commons license and indicate if changes were made.

The images or other third party material in this chapter are included in the chapter's Creative Commons license, unless indicated otherwise in a credit line to the material. If material is not included in the chapter's Creative Commons license and your intended use is not permitted by statutory regulation or exceeds the permitted use, you will need to obtain permission directly from the copyright holder.

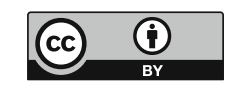

